\title{
Biotransformation of acetophenone to 1-phenylethanol by fungi
}

\author{
Masahiko Nagaki ${ }^{1 *}$, Ryoko Sato ${ }^{2}$, Shingo Tanabe ${ }^{2}$, Takuya Sato ${ }^{2}$, Yuji Hasui ${ }^{1}$, \\ Yukiyasu Chounan ${ }^{3}$, Kazuaki Tanaka ${ }^{4}$, and Yukio Harada ${ }^{4}$ \\ 1 Department of Nursing, School of Health Sciences, Hirosaki University of Health and Welfare, 3-18-1 Sanpinai, \\ Hirosaki, Aomori 036-8102, Japan \\ 2 Graduate School of Science and Technology, Hirosaki University, 3 Bunkyo-cho, Hirosaki, Aomori 036-8561, \\ Japan \\ 3 Faculty of Education, Hirosaki University, 1 Bunkyo-cho, Hirosaki, Aomori 036-8560, Japan \\ 4 Applied Biosciences, Faculty of Agriculture and Life Science, Hirosaki University, 3 Bunkyo-cho, Hirosaki, \\ Aomori 036-8561, Japan \\ * Corresponding author: Fax: 81-172-27-1001 (Ext. 414), and/or e-mail: nagakim@jyoto-gakuen.ac.jp
}

\begin{abstract}
The fungi Botrytis cinerea and Colletotrichum acutatum were used to convert acetophenone to 1-phenylethanol. B. cinerea produced a $98.5 \%$ yield of 1 -phenylethanol with an enantiomeric excess of $93.8 \%$. Conversely, addition of 1-phenylethanol did not change the reaction rate or product composition. In contrast, the reaction catalyzed by $C$. acutatum was markedly slower, commencing only after an approximately three-day delay. The concentration of substrate decreased gradually over approximately 14 days, coinciding with increased production of 1-phenylethanol. Phenol was produced after 18 days, and the final yields of 1-phenylethanol and phenol were $38.1 \%$ and $61.5 \%$, respectively.
\end{abstract}

Key words: biotransformation, Botrytis cinerea, Colletotrichum acutatum, acetophenone, chiral compound

\section{INTRODUCTION}

Because chemical reactions using biocatalysts proceed under "mild" conditions such as atmospheric pressure, room temperature, neutral $\mathrm{pH}$, and in an aqueous solution, they typically do not harm the environment. Cultured microorganisms are therefore frequently used to catalyze reactions such as hydrolysis [1-4], addition [5], substitution [6-9], oxidation [10-13], reduction [14-16], prenylation [17-21], and glycosylation [22-25]. Biotransformations are particularly advantageous compared to organic chemical syntheses because optically active molecules are produced from prochiral substrates, and chiral alcohols are important products of the pharmaceutical industry [26-29].

We previously studied the biotransformation of the prenyl alcohols using cultured pumpkin cells [20]. Several studies were unsuccessful; in one, we evaluated the oxidation of aromatic compounds using apple callus. However, contamination by bacteria occured in the flask, and the reaction occurred from the middle to the opposite direction. It has since returned to raw material. Inspired by this finding, we became interested in determining if the various alcohols could be produced from acids or ketones using a more advantageous system. We reasoned that redox potential might be controlled using a green chemistry target, and after reviewing possible biocatalysts, we found that yeasts are used to synthesize chiral compounds as well as to catalyze various biotransformations. We ultimately decided to use plant pathogens that do not infect humans or animals and are used by a small number of researchers [21, 22]. For this purpose, we consulted Professor Yukio Harada, a specialist in plant pathology at the Faculty of Agriculture and Life Science, Hirosaki University, who provided samples of Botrytis cinerea and Colletotrichum acutatum and instructions for implementing cultivation methods. In particular, $B$. cinerea is indispensable for the manufacture of la pourriture noble, or noble rot [30-32]. Here we assessed the abilities of these two fungal species to catalyze the biotransformation of acetophenone. Incidentally, the research of biotransformation of acetophenone using these fungi as biocatalysts is now for the first time.

\section{EXPERIMENTAL}




\subsection{Analysis}

Reaction products were extracted with ether and measured using high-performance liquid chromatography (HPLC). We used a LichroCART column (Merck-Japan, Tokyo, Japan) eluted with hexane:2-propanol (40:1). To determine the chirality of the products, we used a chiral column (CHIRALPAK AD-H; Daicel Chemical Industries, Ltd., Tokyo, Japan) eluted with hexane:2-propanol (99:1). Gas-chromatography/mass spectrometric (GC-MS) analysis was used to identify the reaction products. The analytical system consisted of a JEOL Q 1000GC Md II Mass Spectrometer (Japan Electron Optics Laboratory Co., Ltd., Tokyo, Japan) equipped with an HP-5 column $(0.32 \mathrm{~mm} \times$ $30 \mathrm{~m} \times 0.25 \mu \mathrm{m})$. Helium was delivered at 1 $\mathrm{mL} / \mathrm{min}$. The temperature gradient started with a 4.7-min hold at $50{ }^{\circ} \mathrm{C}$, increased by $15{ }^{\circ} \mathrm{C} / \mathrm{min}$ for $25 \mathrm{~min}$, and ended with a 2 -min hold at $250{ }^{\circ} \mathrm{C}$. Injector and GC-transfer-line temperatures were $200{ }^{\circ} \mathrm{C}$.

\subsection{Chemicals}

Acetophenone (1st grade) and 1-phenylethanol (special grade) were purchased from Wako Pure Chemical Industries, Ltd. (Osaka, Japan). NADPH was purchased from Orient Yeast Co., Ltd. (Tokyo, Japan); 2-propanol and dimethylsulfoxide from Kanto Chemical Co., Inc. (Tokyo, Japan); and hexane (special grade) from Nacalai Tesque, Inc. (Kyoto, Japan). To determine enantiomeric excess $(\% \quad e e), \quad(S)$ - and $(R)$-1-phenylethanol were purchased from Sigma-Aldrich, Tokyo, Japan.

\subsection{Culture conditions}

Fungi were cultured using potato-dextrose-agar (PDA) E-MF21 (Eiken Chemical Co., Ltd., Tokyo, Japan) or potato-sucrose-agar (PSA) culture medium. Stocks of B. cinerea and C. acutatum (Fig 1 and 2) were prepared by removing a $5-\mathrm{mm}^{2}$ section of agar using a platinum wire and inoculating the section into fresh culture medium. Stocks were stored at $20^{\circ} \mathrm{C}$.

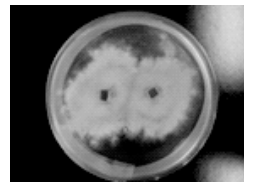

Fig. 1. B. cinerea

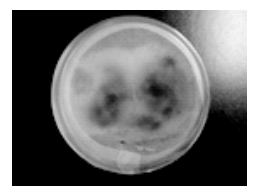

Fig. 2. C. acutatum
2.4. Starter cultures of fungi and biotransformation of acetophenone

Sterile techniques and media were used. The surfaces (hyphae) of colonies of $B$. cinerea and $C$. acutatum were harvested using a platinum wire and transferred to sterile potato-sucrose medium (50 $\mathrm{mL})$ in an Erlenmeyer flask $(100 \mathrm{~mL})$. After the cultures were shaken in the dark at $20{ }^{\circ} \mathrm{C}$ for $72 \mathrm{~h}$, acetophenone or 1-phenylethanol was added, and the flasks were rotated ( 90 rotations $/ \mathrm{min}$ ) using a Taitec NR3 shaker (Taitec, Saitama, Japan).

\subsection{Time course of acetophenone production}

Samples $(1 \mathrm{~mL})$ of cultures were harvested from 0 to $24 \mathrm{~h}$, extracted with ether, and analyzed using HPLC and GC-MS. We determined the effects of adding NADPH $\left(0.50 \times 10^{-3} \mathrm{mmoL}\right)$ to the culture medium.

\section{RESULTS AND DISCUSSION}

3.1. Biotransformation of acetophenone and (rac)-1-phenylethanol by $B$. cinerea

HPLC analysis of culture supernatants of $B$. cinerea detected peaks eluting at $9.4 \mathrm{~min}$ (acetophenone) and $19.5 \mathrm{~min}$. The latter peak was identified as 1-phenylethanol, as expected, compared with the preparation. In contrast, 1-phenylethanol did not detectably react. Although numerous reports have used various biocatalysts to synthesize acetophenone, none has used $B$. cinerea as the biocatalyst [33-42].

3.2. Time course of the biotransformation of acetophenone by $B$. cinerea in the presence of NADPH

We investigated the time course of biotransformation of acetophenone using $B$. cinerea. (rac)-1-Phenylethanol was produced in yields of $69.8 \%, 92.9 \%$, and $97.9 \%$ after 24,48 , and $72 \mathrm{~h}$, respectively (Fig. 3).

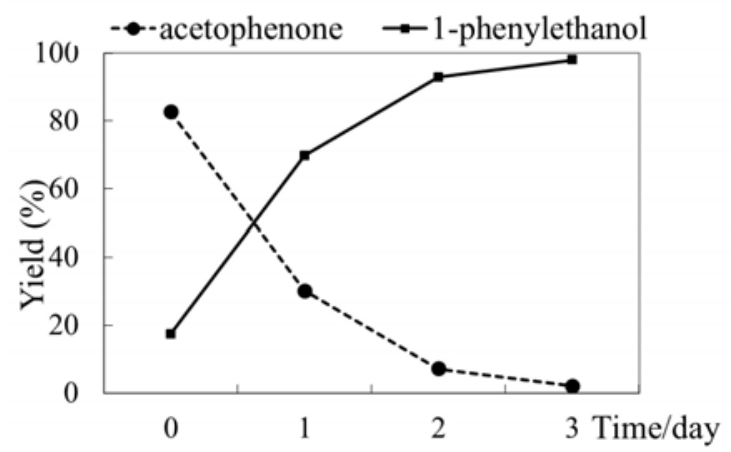

Fig. 3. Time course of biotransformation of acetophenone in the presence of NADPH.

By the measurement of HPLC with chiral column, it was confirmed that acetophenone is converted to efficient $(S)$-1-phenylethanol $(93.8 \%$ ee $)$ (Fig. 4). Although the chirality of the product is influenced by the type of biocatalyst [33-37], the $(S)$-derivative was the main product detected here.

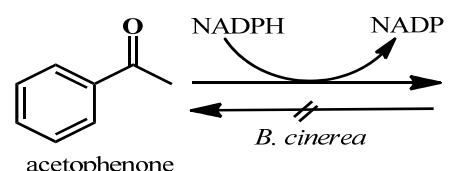<smiles>C[C@H](O)c1ccccc1</smiles>

$(S)$-1-phenylethanol

Fig. 4. Biotransformation of acetophenone by $B$. cinerea. 
3.3. Time course of biotransformation of acetophenone by $C$. acutatum

Compared with $B$. cinerea, biotransformation of acetophenone by cultures of $C$. acutatum started slowly. The acetophenone peak that eluted at 9.4 min from the HPLC column began to decrease gradually from 3 to 12 days, while the 1-phenylethanol peak that eluted at $19.5 \mathrm{~min}$ began to increase. However, after 18 days, a new peak appeared that eluted at $14.3 \mathrm{~min}$, increasing to $56.8 \%$ of the area of the acetophenone peak, and only the 1-phenylethanol and new peak were detectable. Two peaks eluted from the GC column at 6.5 and $8.1 \mathrm{~min}$, and the latter was identified as 1-phenylethanol. The mass spectrum of the new product (GC, $6.5 \mathrm{~min}$ ) showed a molecular ion [M] at $\mathrm{m} / z 94$ (base peak), corresponding to $\mathrm{C}_{6} \mathrm{H}_{6} \mathrm{O}$, together with characteristic fragments at $\mathrm{m} / z 66[\mathrm{M}$ $-28]^{+}$(relative intensity, 28.3) and 65 . We verified that this compound was phenol. The coincidence rate of the GC-MS library data was $92.2 \%$. The time course of the biotransformation of acetophenone by C. acutatum is shown in Fig. 5 .

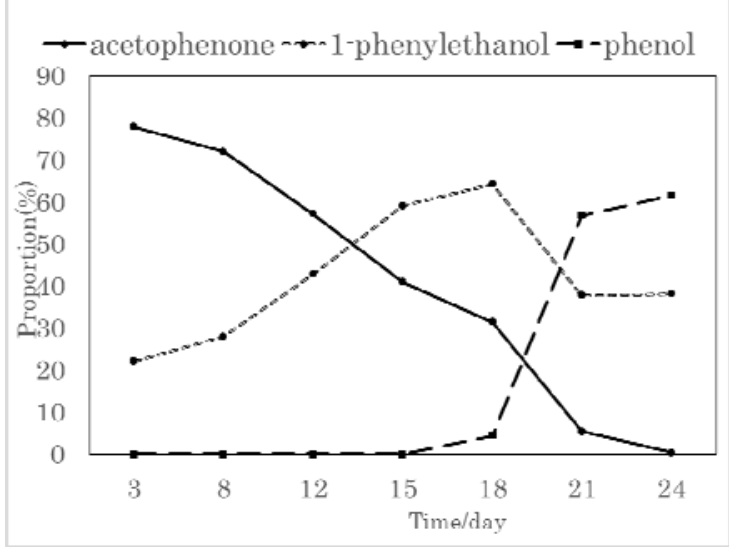

Fig. 5. Time course of the biotransformation of acetophenone by $C$. acutatum

These findings indicate that acetophenone was converted to phenyl acetate through Baeyer-Villiger oxidation [38] (Fig. 6), which occurs frequently during biotransformations [35-44]. The reaction is as shown in Fig. 7.

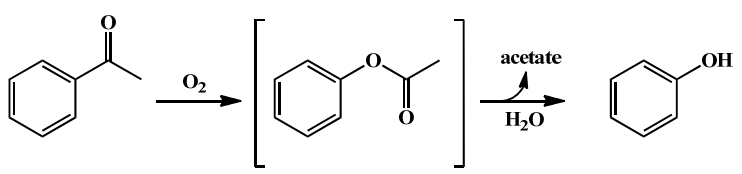

Fig. 6. Predicted biosynthetic pathway of acetophenone.

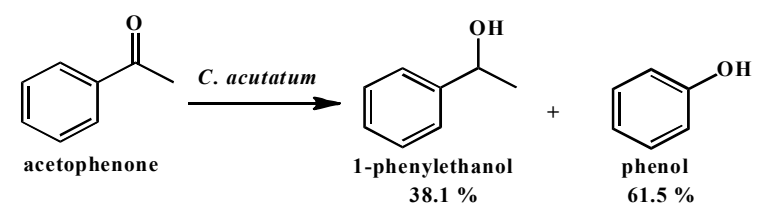

Fig. 7. Biotransformation of acetophenone by $C$. acutatum.

\section{CONCLUSIONS}

$B$ cinerea converted acetophenone to 1 -phenylethanol with a yield of $98.5 \%(93.8 \% e e)$. We conclude that the reaction was catalyzed by an enzyme such as oxidoreductase that required NAPDH as its coenzyme and that the reaction generated the hydride of the nicotinamide group of NADPH that attacked the re-face of the prochiral surface of the substrate (Fig. 8).

In contrast, the biotransformation of acetophenone by $C$. acutatum produced phenol from 1-phenylethanol via phenyl acetate through Baeyer-Villiger oxidation (Fig. 6). The present findings may further improve the yields of beneficial compounds generated using green chemistry.

\section{ACKNOWLEDGMENTS}

This work was supported in part by a grant from the Cosmetology Research Foundation (to M.N.) and in part by the President Specification Research of Hirosaki University of Health and Welfare.

\section{REFERENCES}

[1] E. Brenna, F. Cannavale, M. Crotti, F. Parmeggiani, A. Romagnolo, F. Spina, G. C. Varese, J. Mol. Catal. B: Enzy., 116, 83-88 (2015).

[2] K. Dasu, J. Liu, L. S. Lee, Environ. Sci. Technol., 46(7), 3831-3836 (2012).

[3] V. Andreoni, G. Baggi, S. Bernasconi, C. Foglieni, F. Pelizzoni, Appl. Microbiol. Biotechnol., 33(6), 633-636 (1990).

[4] E. Kreuger, B. Sipos, G. Zacchi, S. Svensson, L. Björnsson, Bioresour.Technol., 102(3), 3457-3465 (2011).

[5] S. J. Brooks, E. M. Doyle, C. Hewage, J. P. G. Malthouse, W. Duetz, K. E. O'Connor, Appl. Microbiol. Biotechnol., 64(4), 486-492 (2004).

[6] J. Huang, G. Ofek, L. Laub, M. K. Louder, N. A. Doria-Rose, N. S. Longo, M. Connors, Nature, 491(7424), 406-412 (2012).

[7] M. Khlupova, I. Vasil'Eva, G. Shumakovich, O. Morozova, V. Chertkov, A. Shestakova, A. Yaropolov, J. Mol. Catal. B: Enzy., 123, 62-66 (2016).

[8] J. Popłoński, S. Sordon, T. Tronina, A. Bartmańska, E. Huszcza, J. Mol. Catal. B: Enzy., 117, 1-6 (2015).

[9] E. Wiltrout, J.M. Goodenbour, M. Fréchin, T. Pan. Nucleic Acids Res., 40(20), 10494-10506 (2012).

[10] D. N. Obanda, T.F. Shupe, Wood Fiber Sci., 41(2), 157-167 (2009).

[11] Y. S. Liu, G. G. Ying, A. Shareef, R. S. Kookana, Water Res., 45(16), 5005-5014 (2011).

[12] S. Wang, C. N. Mulligan, J. Hazard. Mater., 138(3), 459-470 (2006). 


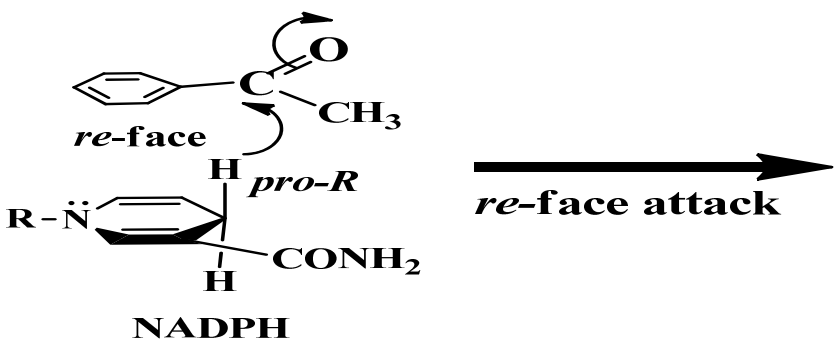

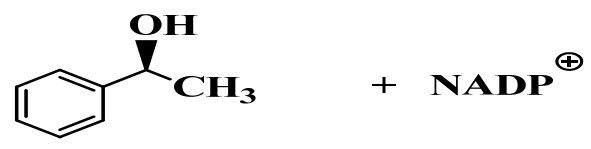

(S)-1-phenylethanol

Fig. 8. Transfer of $\mathrm{H}^{-}($proR $-\mathrm{H})$ from NADPH to acetophenone.

[13] J. S. Yadav, D. L. Lawrence, B. A. Nuck, T. W. Federle, C. A. Reddy, Biodegradation, 12(6), 443-453 (2001).

[14] M. H. W. Ruocco, C. S. Chan, T. E. Hanson, T. M. Church, Geomicrobiol. J., 31(9), 769-778 (2014).

[15] T. Ema, H. Yagasaki, N. Okita, M. Takeda, T. Sakai, Tetrahedron, 62(26), 6143-6149 (2006).

[16] R. Boopathy, M. Wilson, C. F. Kulpa, Water Environ. Res., 65(3), 271-275 (1993).

[17] S. T. Belt, P. Cabedo-Sanz, Org. Geochem., 87, 55-67 (2015).

[18] Q. M. Dudley, A. S. Karim, M. C. Jewett, Biotechnol. J., 10(1), 69-82 (2015).

[19] J. H. Choi, Y. W. Ryu, J. H. Seo, Appl. Microbiol. Biotechnol., 68(1), 9-15 (2005).

[20] M. Nagaki, H. Imaruoka, J. Kawakami, K. Saga, H. Kitahara, H. Sagami, R. Oba, N. Ohya, T. Koyama, J. Mol. Cat. B: Enzy. 47, 33-36 (2007).

[21] M. Nagaki, H. Imaruoka, J. Kawakami, N. Ohya, H. Hamada, H. Sagami, T. Koyama, Trans. Mater. Res. Soc. Jpn., 33, 819-822 (2008).

[22] H. Hamada, K. Shimoda, M. Hamada, H. Hamada, M. Takemoto, Nat. Prod. Commun., 8(7), 907-909 (2013).

[23] H. Hamada, K. Shimoda, N. Kubota, M. Hamada, M. Sugamoto, K. Ishihara, H. Hamada, Nat. Prod. Commun., 8(7), 903-904 (2013).

[24] F. C. Huang, J. Hinkelmann, W. Schwab, J. Biotechnol., 216, 100-109 (2015).

[25] Q. Zhao, M. Wang, D. Xu, Q. Zhang, W. Liu, Nature, 518(7537), 115-119 (2015).

[26] V. Hickmann, A. Kondoh, B. Gabor, M. Alcarazo, A. Fürstner, J. Amer. Chem. Soc., 133(34), 13471-13480 (2011).

[27] L. Li, Y. Wang, L. Zhang, C. Ma, A. Wang, F. Tao, P. Xu, Bioresour. Technol., 115, 111-116 (2012).

[28] D. Muñoz Solano, P. Hoyos, M. J. Hernáiz, A. R. Alcántara, J. M. Sánchez-Montero, Bioresour. Technol., 115, 196-207 (2012).

[29] A. Vandenberghe, I. E. Markó, F. Lucaccioni, S. Lutts, Ind. Crops Prod., 42(1), 380-385 (2013).

[30] P. A. G. Elmer, T. Reglinski, Plant Pathology, 55, 155-177 (2006).

[31] H. Yunome, Y. Zenibayashi, M. Date, Hakkoukagaku kaishi, 59(2), 169-175 (1981).

[32] A. A. Nisiotou, K. Rantsiou, V. Iliopoulos, L. Cocolin, G. J. E. Nychas, Int. J. Microbiol., 145(2-3), 432-436 (2011).
[33] R. D. Patron, J. Aleu, R. Hernández-Galán, I.G. Collado, J. Nat. Prod., 63 (1), 44-47 (2000).

[34] Y. O. Nuñez, I. S. Salabarria, I. G. Collado, R. Hernández-Galán, J. Agric. Food Chem., 54 (20), 7517-7521 (2006).

[35] T. Janeczko, W. Bąkowski, E. Walczak, M. Robak, J. Dmochowska-Gładysz, E. Kostrzewa-Susłow, Ann Microbiol. 65, 1097-1107 (2015).

[36] J. Deng, K. Chen, Z. Yao, J. Lin, D. Wei, J. Mol. Cat. B: Enzy. 118, 1-7 (2015).

[37] K. Nakamura, K. Takenaka, M. Fujii, Y. Ida, Tetrahedron Lett., 43, 3629-3631 (2002).

[38] K. Nakamura, R. Yamanaka, T. Matsuda, T. Harada, Tetrahedron: Asymmetry, 14, 2659-2681 (2003).

[39] T. Matsuda, R. Yamanaka, K. Nakamura, Tetrahedron: Asymmetry, 20, 513-557 (2009).

[40] R. O. Lopes, J. B. Ribeiro, A. S. Miranda, G. V. V. Silva, L. S. M. Miranda, I. C. R. Leal, R.O. M. A. Souza, Tetrahedron 70, 3239-3242 (2014).

[41] M. Fujii, H. Akita, Y. Ida, T. Nakagawa, K. Nakamura, Appl Microbiol Biotechnol, 77, 45-51 (2007).

[42] H. D. Simpson, V. Alphand, R. Furstoss, J. Mol. Catal. B: Enzy., 16,101-108 (2001).

[43] Patel, R. N. Coord. Chem. Rev., 252, 659-701 (2008).

[44] K. B. Borges, W. S. Borges, R. D. Patron, M. T. Pupo, P. S. Bonato, I. G. Collado, Tetrahedron: Asymmetry, 20, 385-397 (2009).

(Received May 3, 2016; Accepted June 28, 2016; Published Online September 1, 2016) 